\title{
Finite Volume Ocean Circulation Model
}

\author{
Peter C. Chu, and Chenwu Fan \\ Department of Oceanography, Naval Postgraduate School, Monterey, CA 93940, USA (chu@nps.navy.mil)
}

\begin{abstract}
A three dimensional finite volume ocean circulation model with a free surface is presented. The basic equations are transformed from differential into integral forms using the hydrostatic and anelastic approximations. The integral equations are solved for finite volumes (rather than grid points) with the flux conservation easily enforced even on arbitrarily meshes. Moreover, this model can easily incorporate the upwind scheme to increase the computational stability and the high-order combine compact schemes to enhance the accuracy. For abrupt topography, a crystal grid discretization is designed to reduce computational errors such that the four lateral boundaries of each finite volume are perpendicular to $x$ and $y$ axes, and the two vertical boundaries are not purely horizontal. This grid system reveals a superior feature than z- and sigma coordinate systems. The accuracy of this model was tested by the standard seamount test case.
\end{abstract}

\section{INTRODUCTION}

Four different schemes are available to solve partial differential equations numerically: (1) spectral or spectral transform, (2) finite difference, (3) finite element, and (4) finite volume. Because of the lateral boundaries with complicated shape, the ocean basin is inherently ill suited to the spectral technique. Due to the flexibility in adapting the grid locally to any desired resolution, the finite element method has been applied to 2D barotropic problems extensively such as tides and storm surge (Foreman et al., 1993; Le Provost et al. 1994), and to 3D baroclinic problems only in the recent years (e.g., Lynch et al., 1996). A principal problem of this method appears to be the mass conservation. While globally this conservation is assured, it may not conserve the mass locally (Kantha and Clayson, 2000).

The finite difference method that transforms the partial differential equations into difference equations at grid points is commonly used in regional and basin-scale ocean modeling. Let $(x, y)$ and $z$ represent the horizontal and vertical directions. Various finite difference models use different vertical coordinates such as $z$-coordinate (e.g., Bryan, 1969), terrain following sigma (e.g., Blumberg and Mellor, 1987) and s-coordinate (Song and Haidvogel, 1994), and isopycnal coordinate (Bleck et al., 1992). The solutions of the finite-difference models are valid only at the grid points. For coastal oceans, the finite-difference models usually use the terrain following sigma coordinate and have large truncation errors at steep topography that is caused by horizontal pressure gradient errors. Much work has been conducted to improve the accuracy of the sigma-coordinate finite- difference models (e.g., Gary, 1973; Beckman and Haidvogel, 1993; McCalpin, 1994; Mellor et al., 1994; Song and Wright, 1998a,b; Chu and Fan, 1997, 1998, 1999, 2000, 2001, 2002).

The finite volume method that transforms the partial differential equations into integral equations at finite volumes has yet been popular in ocean modeling and simulation. However, the conservation is easily enforced even on arbitrary grids because the integral equations link the temporal variability of the dependent variables for the volume to the fluxes across the boundary of that volume (Kobayashi, 1999; Hermeline, 2000). This leads to the volume setup very flexible that makes the finite volume method invaluable especially in the abrupt topography.

In this paper, we present the formulation and preliminary test of the finite volume ocean model (VOM). The outline of this part is as follows: A description of the dynamic and thermodynamic integral equations is given in section 2. A depiction of the crystal finite volume, flux computation, and explicit finite volume scheme is given in sections 3,4 , and 5 . The preliminary model test case is depicted in section 6 . The comparison between the finite difference and finite volume methods is discussed in section 7. In section 8 , the conclusions are presented.

\section{DYNAMIC AND THERMODYNAMIC INTEGRAL EQUATIONS}

The model is established on the base of the two approximation: hydrostatic and anelastic. The anelastic approximation is to assume that the local time rate of change in density $(\rho)$ is small; the continuity equation may be approximated by (Ogura and Phillips, 1962)

$$
\nabla \cdot(\rho \mathbf{V})=0
$$

Here $\mathbf{V}=(u, v, w)$ is the velocity vector and $\nabla$ is the three-dimensional gradient operator. The momentum equation is given by

$$
\frac{\partial(\rho \mathbf{V})}{\partial t}+\nabla \cdot(\rho \mathbf{V V})=-\nabla p+\nabla \cdot(\mu \nabla \mathbf{V})+\mathbf{F}
$$

where $p$ is the pressure, $\mu$ is the eddy viscosity. $\mathbf{F}$ contains Coriolis force and gravity. Let $\phi$ be a scalar 
representing temperature, salinity, satisfying the advection-diffusion equation

$$
\frac{\partial \phi}{\partial t}+\nabla \cdot(\mathbf{V} \phi)=\nabla \cdot\left(\kappa_{\phi} \nabla \phi\right)+F_{\phi}
$$

where $\kappa_{\phi}$ and $F_{\phi}$ are the mixing coefficient and the source term for $\phi$, respectively. The eddy viscosity and vertical mixing coefficients are determined using the level-2 turbulence closure (Mellor and Yamada, 1974).

Integration of (2.1) on a finite volume $\Omega$ (Fig. 1) leads to

$$
\int_{\Omega} \nabla \cdot(\rho \mathbf{V}) d \Omega=\oint_{\Gamma} \rho \mathbf{V} \cdot \mathbf{n} d \Gamma
$$

Integration of (2.2a) and (2.2b) leads to

$$
\begin{aligned}
& \int_{\Omega} \frac{\partial(\rho \mathbf{V})}{\partial t} d \Omega+\oint_{\Gamma} \rho \mathbf{V V} \cdot n d \Gamma \\
& \quad=-\oint_{\Gamma} p d \Gamma+\oint_{\Gamma} \mu \nabla \mathbf{V} \cdot \mathbf{n} d \Gamma+\int_{\Omega} \mathbf{F} d \Omega,
\end{aligned}
$$

and

$$
\begin{aligned}
\int_{\Omega} \frac{\partial \phi}{\partial t} d \Omega+\oint_{\Gamma} \phi \mathbf{V} \cdot n d \Gamma \\
\quad=\oint_{\Gamma} \kappa_{\phi} \nabla \phi \cdot \mathbf{n} d \Gamma+\int_{\Omega} F_{\phi} d \Omega
\end{aligned}
$$

where $\Gamma$ is the boundary of $\Omega$ and $\mathbf{n}$ is the unit vector normal to $\Gamma$ (outward positive).

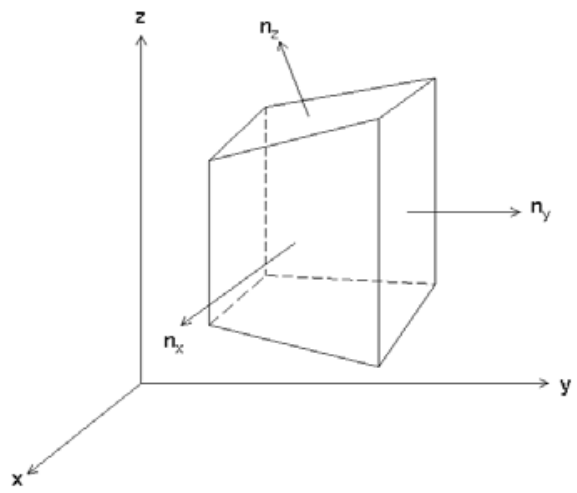

Fig. 1. Description of a finite volume. Here $\left(n_{x}, n_{y}, n_{z}\right)$ are unit vectors normal to the surface of the volume.

The ocean circulation model written in the integral form has big advantage to construct conserved numerical schemes in terrain-following coordinate models. For example, regional oceanic (or atmospheric) prediction models usually use terrain-following sigma coordinates. The water column is divided into the same number of grid cells independence of depth. We restrict attention to two dimensions. Let $(x, z)$ denote Cartesian coordinates and $\left(x^{*}, \sigma\right)$ be the sigma coordinates. The conventional relationship between $z$ - and sigma-coordinates is given by

$$
x=x^{*}, z=\sigma H\left(x^{*}\right),
$$

where $z$ and $\sigma$ increase vertically upward such that $z=$ $\sigma=0$ at the surface and $\sigma=-1, z=-H$ at the bottom. The horizontal pressure gradient can be computed by

$$
\frac{\partial p}{\partial x}=\frac{\partial p^{*}}{\partial x^{*}}-\frac{\sigma}{H} \frac{\partial H}{\partial x} \frac{\partial p}{\partial \sigma} .
$$

Using finite difference schemes, the first term in (2.6) is easily written in a conservation form, but not the second term. This leads to "hydrostatic inconsistency" problem (Mesinger, 1984; Haney, 1991). This problem will be gone when a finite volume ocean circulation model is used.

For a temporally varying finite volume, the time rate of change of the volume-integrated $\phi$-value is computed by

$$
\frac{\partial}{\partial t} \int_{\Omega} \phi d \Omega=\int_{\Omega} \frac{\partial \phi}{\partial t} d \Omega+\phi \frac{\partial \Omega}{\partial t} .
$$

Substitution of (2.7) into (2.4b) leads to

$$
\begin{aligned}
& \frac{\partial}{\partial t} \int_{\Omega} \phi d \Omega-\phi \frac{\partial \Omega}{\partial t}+\oint_{\Gamma} \phi \mathbf{V} \cdot \mathbf{n} d \Gamma \\
& =\oint_{\Gamma} \kappa_{\phi} \nabla \phi \cdot \mathbf{n} d \Gamma+\int_{\Omega} F_{\phi} d \Omega .
\end{aligned}
$$

which is the basic equation for $\phi$ (integral $\phi$-equation). Time integration of (2.8) from $t_{1}$ to $t_{2}$ gives

$$
\begin{aligned}
& \int_{\Omega} \phi\left(t_{2}\right) d \Omega-\int_{\Omega} \phi\left(t_{1}\right) d \Omega=-\Delta t \oint_{\Gamma} \phi(\hat{t}) \mathbf{V} \cdot \mathbf{n} d \Gamma \\
& +\Delta t \oint_{\Gamma} \kappa_{\phi} \nabla \phi(\hat{t}) \cdot \mathbf{n} d \Gamma+\Delta t \int_{\Omega} F_{\phi}(\hat{t}) d \Omega+\Delta t\left(\phi \frac{\partial \Omega}{\partial t}\right)_{\hat{t}}
\end{aligned}
$$

where $\Delta t=t_{2}-t_{1}$, and $t_{1} \leq \hat{t} \leq t_{2}$. If $\hat{t}=t_{1}$, the scheme is explicit; if $\hat{t}=t_{2}$, the scheme is implicit. To adjust $\hat{t}$ may lead to a high-order temporal discrete scheme. The last term in the right-hand side of (2.9) represents the temporal volume change due to surface elevation fluctuation. Similarly, the time integration of (2.4a) can also be obtained.

\section{CRYSTAL VOLUME}

Let $\left(\mathbf{e}_{x}, \mathbf{e}_{y}\right)$ and $\mathbf{e}_{z}$ be the constant unit vectors in the horizontal and vertical, respectively. In VOM, the integration is over the boundary of a volume. The unit vectors for each finite volume may not be constant. The discretization is designed such that the four lateral boundaries are perpendicular to $\mathbf{e}_{x}$ or $\mathbf{e}_{y}$ (Fig. 1), 


$$
\mathbf{n}_{x}=\mathbf{e}_{x}, \quad \mathbf{n}_{y}=\mathbf{e}_{y}
$$

and the two vertical boundaries are not horizontal, and the normal unit vector on the vertical boundaries of a volume $\left(\mathbf{n}_{z}\right)$ is determined by

$\mathbf{n}_{z}=\left(-z_{x} \mathbf{n}_{x}-z_{y} \mathbf{n}_{y}+\mathbf{e}_{z}\right) / \sqrt{\left(z_{x}\right)^{2}+\left(z_{y}\right)^{2}+1}$.

where

$$
z_{x}=\frac{\partial z}{\partial x}, \quad z_{y}=\frac{\partial z}{\partial y} .
$$

Each finite volume looks like a crystal (Fig. 1), and therefore this scheme is named the finite crystal volume scheme.

Since the ocean surface changes with the time, any finite crystal volume also varies with time. The discretization in the horizontal directions

$$
x=x(i), \quad y=y(j)
$$

is the same as the $z$-coordinate system, and vertical discretization

$$
z=z(i, j, k, t)
$$

varies with the location $(i, j)$ and time $(\mathrm{t})$. To determine $\mathbf{n}_{z}$ in a finite crystal volume enclosed by $\left(x_{\mathrm{i}}, \mathrm{x}_{\mathrm{i}+1}\right)$ in the $x$ direction and by $\left(y_{\mathrm{j}}, y_{\mathrm{j}+1}\right)$ in the $y$ direction, a bi-linear interpolation is used to obtain the $z$-boundary for that crystal volume,

$$
\begin{aligned}
& z(x, y)=z_{11} \frac{\left(x-x_{i+1}\right)\left(y-y_{j+1}\right)}{\left(x_{i}-x_{i+1}\right)\left(y_{j}-y_{j+1}\right)}+z_{12} \frac{\left(x-x_{i+1}\right)\left(y-y_{j}\right)}{\left(x_{i}-x_{i+1}\right)\left(y_{j+1}-y_{j}\right)} \\
& +z_{21} \frac{\left(x-x_{i}\right)\left(y-y_{j+1}\right)}{\left(x_{i+1}-x_{i}\right)\left(y_{j}-y_{j+1}\right)}+z_{22} \frac{\left(x-x_{i}\right)\left(y-y_{j}\right)}{\left(x_{i+1}-x_{i}\right)\left(y_{j+1}-y_{j}\right)}
\end{aligned}
$$

where

$$
\begin{gathered}
z_{11}=z\left(x_{i,} y_{j}\right), z_{12}=z\left(x_{i,} y_{j+1}\right), \\
z_{21}=z\left(x_{i+1}, y_{j}\right), z_{22}=z\left(x_{i+1}, y_{j+1}\right) .
\end{gathered}
$$

Substitution of (3.6) into (3.2) determines the unit vector $\mathbf{n}_{z}$ at $\left(x_{i+1 / 2}, y_{j+1 / 2}\right)$,

$$
\begin{aligned}
& \cos \theta_{x}=-\frac{z_{x}\left(x_{i+1 / 2}, y_{j+1 / 2}\right)}{\sqrt{1+z_{x}^{2}+z_{y}^{2}}}, \quad \cos \theta_{y}=-\frac{z_{y}\left(x_{i+1 / 2}, y_{j+1 / 2}\right)}{\sqrt{1+z_{x}^{2}+z_{y}^{2}}} \\
& \cos \theta_{z}=\frac{1}{\sqrt{1+z_{x}^{2}+z_{y}^{2}}},
\end{aligned}
$$

where

$$
x_{i+1 / 2}=\left(x_{i}+x_{i+1}\right) / 2, \quad \mathrm{y}_{j+1 / 2}=\left(y_{j}+y_{j+1}\right) / 2
$$

are the horizontal coordinates of the center of the horizontal cell.

Comparison among the crystal, z- and sigma-grid systems around a seamount (Fig. 2) shows the least distortion in the crystal system. The normal gradient of any variable $\phi$ is calculated by

$$
\frac{\partial \phi}{\partial n_{z}}=\nabla \phi \cdot \mathbf{n}_{z}=\frac{\frac{\partial \phi}{\partial z}-\frac{\partial \phi}{\partial x} z_{x}-\frac{\partial \phi}{\partial y} z_{y}}{\sqrt{\left(z_{x}\right)^{2}+\left(z_{y}\right)^{2}+1}}
$$
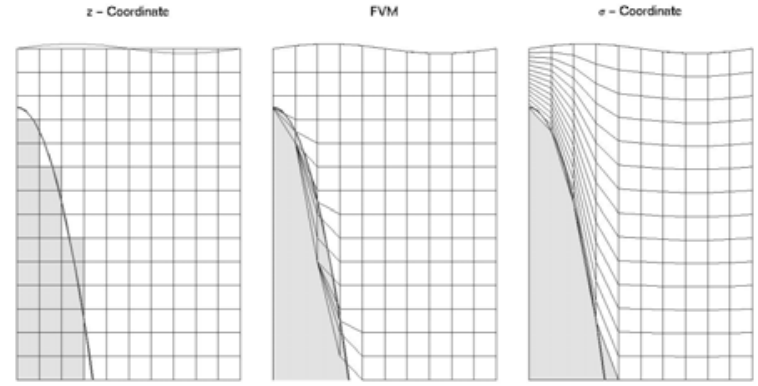

Fig. 2. Comparison among z-coordinate, sigma-coordinate, and finite volume systems.

\section{FLUX COMPUTATION}

The surfaces perpendicular to $\left(\mathbf{n}_{x}, \mathbf{n}_{y}, \mathbf{n}_{z}\right)$ are called the $x$-, $y$ - and $z$-boundaries, respectively. In the basic equations (2.4a) and (2.4b), various fluxes across the boundaries of the finite crystal volume need to be computed. For the $x$-boundary, the fluxes of mass and tracer $\phi$ are computed by

$$
\begin{aligned}
f_{m}^{x} & =\int_{x-\text { boundary }} \rho \mathbf{V} \cdot \mathbf{n}_{x} d \Gamma=\overline{\rho u} \Delta S_{y z}, \\
f_{\phi}^{x} & =\int_{x-\text { boundary }} \phi \mathbf{V} \cdot \mathbf{n}_{x} d \Gamma=\overline{\phi u} \Delta S_{y z} ;
\end{aligned}
$$

the turbulent flux and pressure forces are computed by

$$
\begin{gathered}
f_{\phi}^{x}=\int_{x \text {-boundary }} \phi \mathbf{V} \cdot \mathbf{n}_{x} d \Gamma=\overline{\phi u} \Delta S_{y z}, \\
P_{x}^{x}=p \Delta S_{y z}, \quad P_{y}^{x}=0
\end{gathered}
$$

where $\Delta S_{y z}$ is the area of the trapezoid of the $x$ boundary.

For the $y$-boundary, the fluxes of mass and tracer $\phi$ are computed by

$$
\begin{aligned}
& f_{m}^{y}=\int_{y \text {-boundary }} \rho \mathbf{V} \cdot \mathbf{n}_{y} d \Gamma=\overline{\rho v} \Delta S_{z x}, \\
& f_{\phi}^{y}=\int_{y \text {-boundary }} \phi \mathbf{V} \cdot \mathbf{n}_{y} d \Gamma=\overline{\phi v} \Delta S_{z x} ;
\end{aligned}
$$

the turbulent flux and pressure forces are computed by

$$
f_{\phi}^{y}=\int_{y \text {-boundary }} \phi \mathbf{V} \cdot \mathbf{n}_{y} d \Gamma=\overline{\phi v} \Delta S_{z x},
$$




$$
P_{y}^{y}=p \Delta S_{z x}, P_{y}^{x}=0,
$$

where $\Delta S_{z x}$ is the area of the trapezoid of the yboundary.

For the z-boundary, we have

$$
\begin{gathered}
\left.f_{m}^{z}=\int_{z \text {-boundary }} \rho \mathbf{V} \cdot \mathbf{n}_{z} d \Gamma=\overline{\rho\left(w-u z_{x}-v z_{y}\right.}\right) \Delta S_{x y}, \\
\left.f_{\phi}^{z}=\int_{z \text {-boundary }} \phi \mathbf{V} \cdot \mathbf{n}_{z} d \Gamma=\overline{\phi\left(w-u z_{x}-v z_{y}\right.}\right) \Delta S_{x y}, \\
\left.f_{\tau}^{z}=\int_{z \text {-boundary }} \kappa_{\phi} \nabla \phi \cdot \mathbf{n}_{z} d \Gamma=\overline{\kappa_{\phi}\left(\phi_{z}-\phi_{x} z_{x}-\phi_{y} z_{y}\right.}\right) \Delta S_{x y}, \\
P_{x}^{z}=-p z_{x} \Delta S_{x y}, P_{y}^{z}=-p z_{y} \Delta S_{x y},
\end{gathered}
$$

where $\Delta S_{x y}$ is the area of the trapezoid of the $z$ boundary. by

It is noticed that the derivatives should be computed

$$
\begin{aligned}
& \phi_{x}=\frac{\delta \phi}{\delta x}-z_{x} \frac{\delta \phi}{\delta z}, \phi_{y}=\frac{\delta \phi}{\delta y}-z_{y} \frac{\delta \phi}{\delta z}, \\
& \phi_{z}=\frac{\delta \phi}{\delta z}
\end{aligned}
$$

where $\delta \phi / \delta x, \delta \phi / \delta y$, and $\delta \phi / \delta z$ are computed directly from the grid point data.

\section{EXPLICIT FINITE VOLUME SCHEME}

The finite volume scheme for solving continuity equation (3) at $(i, \mathrm{j}, k)$ is

$$
\begin{aligned}
& f_{m}^{x}\left(i+\frac{1}{2}, j, k\right)-f_{m}^{x}\left(i-\frac{1}{2}, j, k\right) \\
& +f_{m}^{y}\left(i, j+\frac{1}{2}, k\right)-f_{m}^{x}\left(i, j-\frac{1}{2}, k\right) \\
& +f_{m}^{z}\left(i, j, k+\frac{1}{2}\right)-f_{m}^{x}\left(i, j, k-\frac{1}{2}\right)=0 .
\end{aligned}
$$

If $\hat{t}=t_{1}$ in (2.9), the finite volume scheme is explicit. The scheme for solving $(2.9)$ at $(i, j, k)$ is given by

$$
\begin{aligned}
& \left(\phi_{i, j, k}^{n+1}-\phi_{i, j, k}^{n}\right) \Omega_{i, j, k}=f_{\phi}^{x}\left(i-\frac{1}{2}, j, k\right)-f_{\phi}^{x}\left(i+\frac{1}{2}, j, k\right) \\
+ & f_{\phi}^{y}\left(i, j-\frac{1}{2}, k\right)-f_{\phi}^{y}\left(i, j+\frac{1}{2}, k\right) \\
+ & f_{\phi}^{z}\left(i, j, k-\frac{1}{2}\right)-f_{\phi}^{z}\left(i, j, k+\frac{1}{2}\right) \\
+ & f_{\tau}^{x}\left(i-\frac{1}{2}, j, k\right)-f_{\tau}^{x}\left(i+\frac{1}{2}, j, k\right) \\
+ & f_{\tau}^{y}\left(i, j-\frac{1}{2}, k\right)-f_{\tau}^{y}\left(i, j+\frac{1}{2}, k\right) \\
+ & f_{\tau}^{z}\left(i, j, k-\frac{1}{2}\right)-f_{\tau}^{z}\left(i, j, k+\frac{1}{2}\right)
\end{aligned}
$$

$$
+F(i, j, k) \Omega_{i, j, k}+\Delta t\left(\phi \frac{\partial \Omega}{\partial t}\right)_{i, j, k}^{n} .
$$

For $\phi=\rho u$, the pressure gradient force can be separated from the forcing term, i.e., the following term $\left(P_{\text {xterm }}\right)$ should be added into the right-hand side if the forcing term $\mathrm{F}(i, j, k)$ does not include the pressure gradient force,

$$
\begin{aligned}
& P_{x t e r m}=P_{x}^{x}\left(i-\frac{1}{2}, j, k\right)-P_{x}^{x}\left(i+\frac{1}{2}, j, k\right) \\
& +P_{x}^{z}\left(i, j, k-\frac{1}{2}\right)-P_{x}^{z}\left(i, j, k+\frac{1}{2}\right) .
\end{aligned}
$$

For $\phi=\rho v$, it should be

$$
\begin{gathered}
P_{y \text { term }}=P_{y}^{y}\left(i, j-\frac{1}{2}, k\right)-P_{y}^{y}\left(i, j+\frac{1}{2}, k\right) \\
+P_{y}^{z}\left(i, j, k-\frac{1}{2}\right)-P_{y}^{z}\left(i, j, k+\frac{1}{2}\right) .
\end{gathered}
$$

\section{PRELIMINARY TEST}

Usually, verification of a new numerical model should be divided into stages: (1) evaluating its own performance, and (2) identifying its difference from the existing models. Theoretically, the performance of any numerical ocean model should only be tested against analytical or known solutions. Coastal ocean hardly has any analytical solutions. Known solutions become important. Without atmospheric and lateral forcing, the ocean that is initially at rest should be at rest forever. Thus, we have the known solution $(\mathbf{V}=0)$. The seamount test case (Beckmann and Haidvogel, 1993) is to use this known solution for model evaluation.

Several advanced test cases have been proposed to identify the model-model difference and the sensitivity to the choice of (say) advection algorithm, such as gravitational adjustment of density front, residual circulation over a coastal canyon, combined effects of topography and stratification (Haidvogel and Beckmann, 1998). The second stage test should be conducted after the first stage test. In this paper, we only present the first stage evaluation using the seamount test case.

Since the horizontal pressure gradient error is a key issue in the sigma coordinate finite difference models, it is the first step to evaluate the value-added of the finite volume scheme for the horizontal pressure gradient. The Princeton Ocean Model (POM, Blumberg and Mellor, 1987) is implemented for the seamount test case. Two experiments were conducted: (1) conventional POM (i.e., POM with the pressure gradient computed using the finite difference scheme), and (2) POM with the pressure gradient computed using the finite volume scheme. Both finite volume and finite difference schemes are secondorder.

\subsection{Description}


Suppose a seamount to be located inside a periodic $f$ plane $\left(f_{0}=10^{-4} \mathrm{~s}^{-1}\right)$ channel with two solid, free-slip boundaries along constant $y$. Unforced flow over seamount in the presence of resting, level isopycnals is an idea test case for the assessment of pressure gradient errors in simulating stratified flow over topography. The flow is assumed to be reentrant (periodic) in the along channel coordinate (i.e., $x$-axis). This seamount case is chosen to test the performance of VOM. The time steps for barotropic and baroclinic modes are $6 \mathrm{~s}$ and $180 \mathrm{~s}$, respectively. Variable horizontal grid is used with high resolution over the seamount,

$$
\begin{aligned}
& (\Delta x)_{i}=8 \mathrm{~km}\left[1-0.5 \sin \left(\frac{i \pi}{M_{x}}\right)\right], i=1,2, \ldots, M_{x} \\
& (\Delta y)_{j}=8 \mathrm{~km}\left[1-0.5 \sin \left(\frac{j \pi}{M_{y}}\right)\right], \mathrm{j}=1,2, \ldots, M_{y}
\end{aligned}
$$

where $M_{x}=M_{y}=64$. The horizontal diffusivities are using the Smagorinsky form with the coefficient chosen to be 0.2 for this application.

\subsection{Topography}

The domain is a periodic channel, $300 \mathrm{~km}$ long and $300 \mathrm{~km}$ wide. The channel has a far-field depth $\mathrm{h}_{\max }$ and in the center includes an isolated Gaussian-shape seamount with a width $L$ and an amplitude $\mathrm{h}_{\mathrm{s}}$ (Fig. 3),

$$
h(x, y)=h_{\max }\left\{1-0.9 \exp \left[-\frac{\left(x-x_{0}\right)^{2}+\left(y-y_{0}\right)^{2}}{L^{2}}\right]\right\}
$$

where $\left(x_{0}, y_{0}\right)$ are the longitude and latitude of the seamount center. The far-field depth $\left(h_{\max }\right)$ is fixed as $4,500 \mathrm{~m}$. The lateral scale of the seamount $(L)$ is set as $25 \mathrm{~km}$ for the study.

\subsection{Initial Fields}

Suppose that the background fluid is at rest and with a constant salinity (35 ppt) and an exponentially stratified initial temperature (unit: ${ }^{\circ} \mathrm{C}$ )

$T(z)=5+15 \exp \left(\frac{z}{H_{T}}\right)$,

where $H_{T}=1000 \mathrm{~m}$. Since the fluid is initially at rest and the density field is independent on $x$ and $y$, without forcing the velocity and horizontal pressure gradient should be zero. Any nonzero velocities are computational errors.

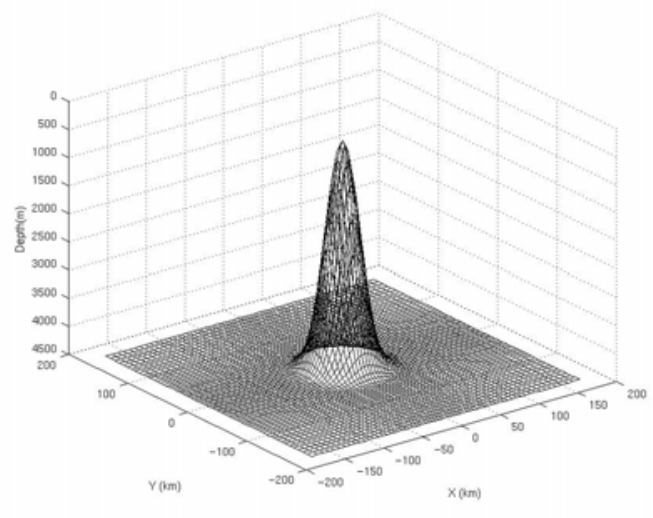

Fig. 3. Seamount geometry.

7. COMPARISON BETWEEN FINITE DIFFERENCE AND FINITE VOLUME SCHEMES

\subsection{Temporal Variations of Error Volume Transport}

Both cases are integrated for 20 days for the standard test. Figure 4 displays errors in the streamfunction after performing $5,10,15$, and 20 days of integration using the finite volume and finite difference schemes. The volume transport streamfunction has a large-scale eightlobe pattern centered on the seamount. The errors in the volume transport reduce more than $50 \%$ from finite difference to finite volume schemes. For example, on the 20th day, the errors in the volume transport varies from 56 to $84 \times 10^{-3} \mathrm{~Sv}$ using the finite difference scheme and from -28 to $45.5 \times 10^{-3} \mathrm{~Sv}$ using the finite volume scheme.

\subsection{Temporal Variations of Pressure Gradient Error}

Owing to a very large number of calculations performed, we discuss the results exclusively in terms of the maximum and spatially averaged absolute values of the horizontal pressure gradient errors, called the maximum pressure gradient error $\left(\mathrm{PG}_{\max }\right)$ and the mean pressure gradient error $\left(\mathrm{PG}_{\mathrm{m}}\right)$. Figure $5 \mathrm{a}$ and $5 \mathrm{~b}$ show the time evolution of $\mathrm{PG}_{\max }$ and $\mathrm{PG}_{\mathrm{m}}$ for the first 20 days of integration using the finite difference and finite volume schemes. Both errors increase with time, however, they are 10-15 times smaller using the finite volume scheme than using the finite difference scheme. For example, at Day-10, $\mathrm{PG}_{\max }=33.42 \times 10^{-9} \mathrm{~N} / \mathrm{m}^{3}$ using the finite difference scheme and $\mathrm{PG}_{\max }=2.16 \times 10^{-9} \mathrm{~N} / \mathrm{m}^{3}$ using the finite volume scheme; $\mathrm{PG}_{\mathrm{m}}=0.449 \times 10^{-9} \mathrm{~N} / \mathrm{m}^{3}$ using the finite difference scheme and $\mathrm{PG}_{\mathrm{m}}=0.04 \times 10^{-9} \mathrm{~N} / \mathrm{m}^{3}$ using the finite volume scheme; at Day-20, $\mathrm{PG}_{\max }=$ $58.41 \times 10^{-9} \mathrm{~N} / \mathrm{m}^{3}$ using the finite difference scheme and $\mathrm{PG}_{\text {max }}=4.18 \times 10^{-9} \mathrm{~N} / \mathrm{m}^{3}$ using the finite volume scheme; $\quad \mathrm{PG}_{\mathrm{m}}=1.596 \times 10^{-9} \mathrm{~N} / \mathrm{m}^{3}$ using the finite difference scheme and $\mathrm{PG}_{\mathrm{m}}=0.150 \times 10^{-9} \mathrm{~N} / \mathrm{m}^{3}$ using the finite volume scheme. 

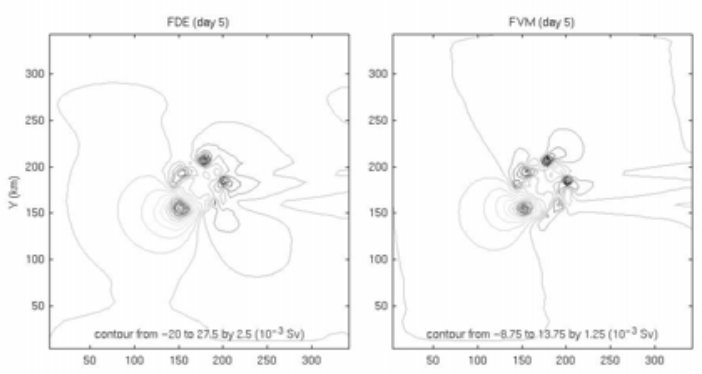

FDE (day 10)
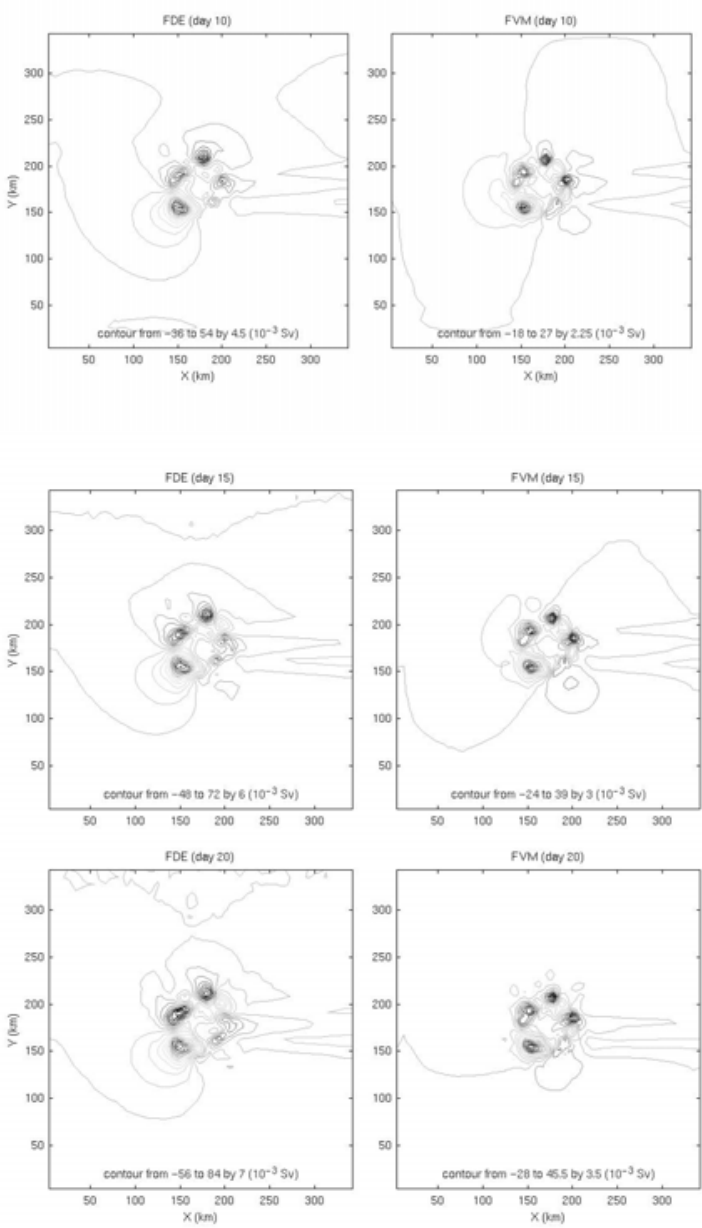

Fig. 4. Volume transport streamfunction (Sv) at day-5, -10, -15 , and -20 using the finite difference and finite volume schemes.

\subsection{Temporal Variations of Error Velocity}

Owing to a very large number of calculations performed, we discuss the results exclusively in terms of the maximum and spatially averaged absolute values of the spurious velocity generated by the pressure gradient errors, called the peak error velocity $\left(\mathrm{V}_{\mathrm{p}}\right)$ and the mean error velocity $\left(\mathrm{V}_{\mathrm{m}}\right)$. Figures $5 \mathrm{c}$ and $5 \mathrm{~d}$ shows the time evolution of the mean and peak error velocity for the first 20 days of integration using the finite difference and finite volume schemes. Both peak and mean error velocities increase with time, however, they are 4 times smaller using the finite volume scheme than using the finite difference scheme. For example, at Day-10, $\mathrm{V}_{\mathrm{p}}=$
$2.61 \mathrm{~cm} / \mathrm{s}$ using the finite difference scheme and $\mathrm{V}_{\mathrm{p}}=$ $0.57 \mathrm{~cm} / \mathrm{s}$ using the finite volume scheme; $\mathrm{V}_{\mathrm{m}}=0.054$ $\mathrm{cm} / \mathrm{s}$ using the finite difference scheme and $\mathrm{V}_{\mathrm{m}}=0.015 \$$ $\mathrm{cm} / \mathrm{s}$ using the finite volume scheme; at Day-20, $\mathrm{V}_{\mathrm{p}}=$ $4.25 \mathrm{~cm} / \mathrm{s}$ using the finite difference scheme and $\mathrm{V}_{\mathrm{p}}=$ $0.98 \mathrm{~cm} / \mathrm{s}$ using the finite volume scheme; $\mathrm{V}_{\mathrm{m}}=1.033$ $\mathrm{cm} / \mathrm{s}$ using the finite difference scheme and $\mathrm{V}_{\mathrm{m}}=0.028$ $\mathrm{cm} / \mathrm{s}$ using the finite volume scheme.

\section{SENSITIVITY STUDY ON TOPOGRAPHY}

One of the difficult problems in shallow water modeling is the uncertainty of the open boundary condition $(\mathrm{OBC})$. At open boundaries where the numerical grid ends, the fluid motion should be unrestricted. Ideal open boundaries are transparent to motions. Two approaches, local-type and inverse-type, are available for determining OBC (Chu and Fan, 2001) with low-order conditions. Before converting any ocean model from second-order scheme to sixth-order scheme, it is important to verify if a high-order interior plus loworder boundary conditions (such as open boundary conditions) would degrade the interior solution. Consider a horizontally homogeneous and stably stratified coastal ocean with a longitudinal and straight coastline and three open boundaries in the south $\left(20^{\circ} \mathrm{N}\right)$, the north $\left(33.5^{\circ} \mathrm{N}\right)$, and the east $\left(x=x_{\mathrm{E}}=320 \mathrm{~km}\right)$. Choose coordinates so that the $y$-axis coincides with the coast, positive $x$ pointing offshore.
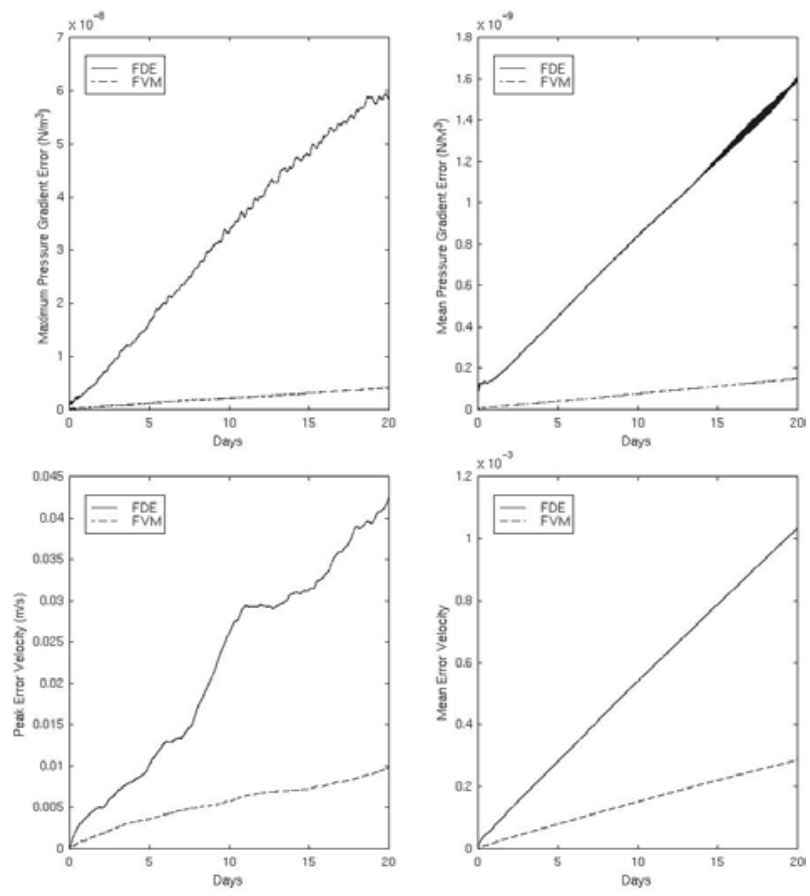

Fig. 5. Comparison between the finite difference and finite volume schemes on temporal variations of (a) maximum pressure gradient error $\left(\mathrm{N} / \mathrm{m}^{3}\right)$, (b) mean pressure gradient error $\left(\mathrm{N} / \mathrm{m}^{3}\right)$, (c) peak error velocity $(\mathrm{m} / \mathrm{s})$, and (d) mean error velocity $(\mathrm{m} / \mathrm{s})$. 
The coastal water is divided into three parts (Fig. 6): shelf ( $\left.0 \leq x \leq x_{0}\right)$, slope $\left(x_{0}<x \leq x_{1}\right)$, and deep water $\left(x_{1}<x \leq x_{E}\right)$. Here, the water depth $(h)$ is only a function of offshore distance $x$. Analytical bottom topography (Fig. 6) is proposed such that shelf and slope are arcs of two circles. The shelf has a smaller radius $(r)$, and the slope has a larger radius $(R)$. The two arcs are connected in such a way that tangent of the bottom topography, $\mathrm{d} h / \mathrm{d} x$, is continuous at the shelf break ( $x=$ $\left.x_{0}\right)$. This requirement is met using the same expanding angle ( $\theta$, maximum slope angle) for both arcs, which is determined by

$$
(1-\cos \theta)=H /(r+R)
$$

Off-shore distance $(x)$, vertical coordinate $(z)$, and water depth $(h)$, and $(r, R)$ are non-dimensionalized by $\hat{x}=\frac{x}{H}, \hat{z}=\frac{z}{H}, \hat{h}(\hat{x})=\frac{h}{H}, \hat{r}=\frac{r}{H}, \hat{R}=\frac{R}{H}$.

The analytical bottom topography is given by,

$$
\hat{h}(\hat{x})= \begin{cases}\hat{r}-\sqrt{\hat{r}^{2}-\hat{x}^{2}}, & \text { if } \hat{x} \leq \hat{x}_{0} \\ 1-\hat{R}+\sqrt{\hat{R}^{2}-\left(\hat{x}_{1}-\hat{x}\right)^{2}}, & \text { if } \hat{x}_{0}<\hat{x} \leq \hat{x}_{1} \\ 1, & \text { if } \hat{x}>\hat{x}_{1}\end{cases}
$$

Using the shelf-to-slope ratio, $k=r / R$, the nondimensional parameters in (8.3) are given by

$$
\begin{aligned}
& \hat{r}=\frac{k}{(1+k)(1-\cos \theta)}, \quad \hat{R}=\frac{1}{(1+k)(1-\cos \theta)}, \\
& \hat{x}_{0}=\hat{r} \sin \theta=\frac{k \cdot \sin \theta}{(1+k)(1-\cos \theta)}, \\
& \hat{x}_{1}=(\hat{r}+\hat{R}) \cdot \sin \theta=\frac{\sin \theta}{1-\cos \theta} .
\end{aligned}
$$

Thus, the nondimensional topography (8.3) has 2 degrees of freedom $(k, \theta)$. The larger the angle $\theta$, the steeper the topography is; the large the value of $k$, the longer the shelf is. Various types of topography (Fig. 7) can be modeled with changing values of $(k, \theta)$. In this study, $\theta$ varies from $5^{\circ}$ to $90^{\circ}$ and $\mathrm{k}$ changes from 0.05 to 1.0 .

For a horizontally uniform density field,

$$
\rho=1025 \mathrm{~kg} / \mathrm{m}^{3}\left[1+0.005\left(1-e^{2 z / H}\right)\right],
$$

the horizontal pressure gradient should be zero. Any nonzero values of the horizontal pressure gradient are computational errors. Figure 8 shows that the finitevolume integration scheme (denoted by HPI scheme) has much smaller errors than the finite difference schemes. It is also noted that the pressure gradient error increases rapidly as $\theta$ increase, and decrease slowly as $k$ increase.

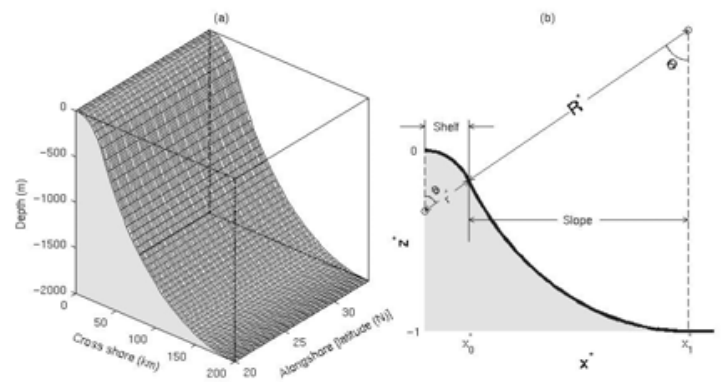

Fig. 6. Coastal geometry with open boundaries.
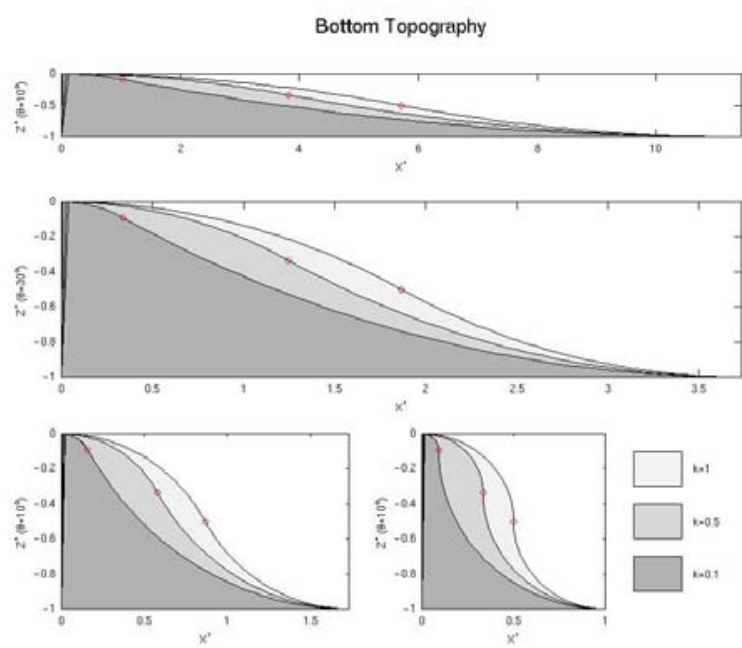

Fig. 7. Topography with various values of $(k, \theta)$.

\section{CONCLUSIONS}

(1) A three-dimensional, finite volume ocean circulation model has been developed. The basic equations are transformed from differential into integral forms using the hydrostatic and anelastic approximations and solved for finite volumes (rather than grid points) with the flux conservation enforced on arbitrarily meshes. This model has great flexibility in establishing model grids.

(2) A crystal grid discretization is proposed such that the four lateral surfaces are perpendicular to two horizontal $(x, y)$ axes; and the two surfaces in the vertical follow the ocean surface and bottom. Such a grid system reveals a superior feature than both $\mathrm{z}$ - and sigma-coordinate systems over abrupt bottom topography.

(3) Seamount test case is the first step to show the valueadded of using finite volume scheme. The finite volume 
scheme leads to a drastic error reduction comparing to the second-order finite difference scheme using POM.

(4) It is noticed that the seamount test case presented here is preliminary. More cases should be conducted in the future for testing the difference between VOM and the existing ocean models using gravitational adjustment of a density front, residual circulation over a coastal canyon, and combined effects of topography and stratification.

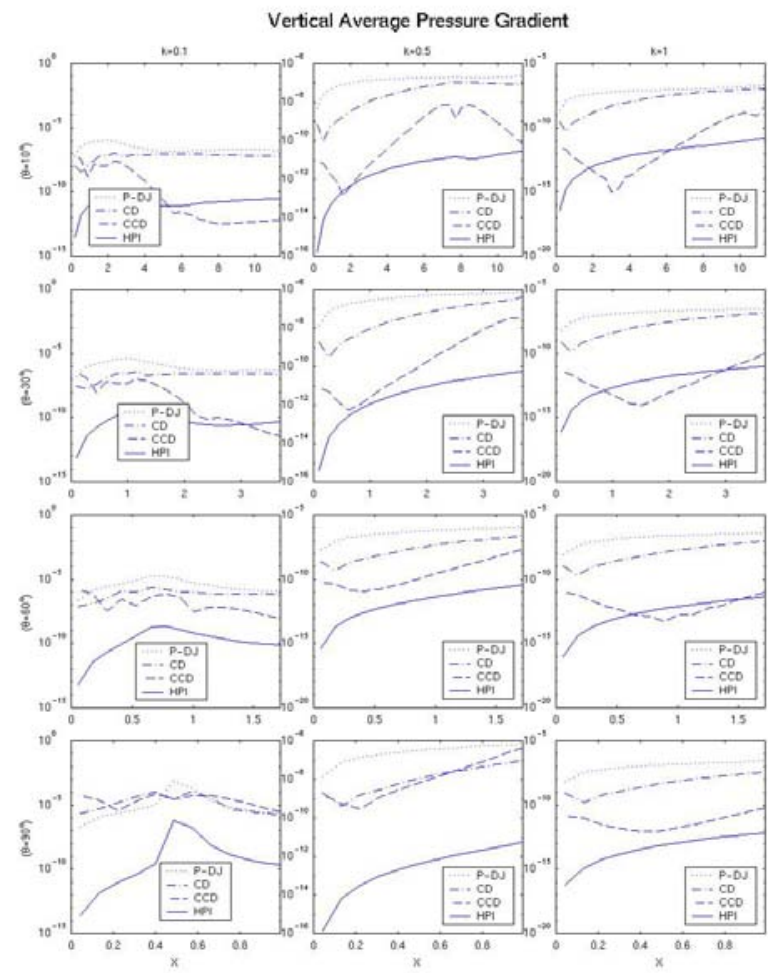

Fig. 8. Comparison between finite-volume scheme (HPI) and three finite difference schemes (P-DJ, CD, and CCD) for various $\mathrm{k}$ and $\theta$.

\section{ACKNOWLEDGMENTS}

This work was funded by the Office of Naval Research, the Naval Oceanographic Office, and the Naval Postgraduate School.

\section{REFERENCES}

[1] Bleck, R., C. Roothe, D. Hu, and L. Smith, 1992: Salinitydriven thermocline transients in a wind- and thermohalineforced isopycnal coordinate model of the North Atlantic. J. Phys. Oceanogr., 22, 1486-1505.

[2] Blumberg, A.F., and G.L. Mellor, 1987: A description of a three-dimensional coastal ocean circulation model. In: ThreeDimensional Coastal Ocean Models (edited by N. Heaps), pp. 1-16, Amer. Geophys. Union, Washington D.C.

[3] Bryan, K., 1969: A numerical model for the study of the circulation of the world oceans. J. Comput. Phys., 4, 347-359.
[4] Chu, P.C., and C.W. Fan, 1997: Sixth-order difference scheme for sigma-coordinate ocean models. J. Phys. Oceanogr, 27, 2064-2071.

[5] Chu, P.C., and C.W. Fan, 1998: A three-point combined compact difference scheme. J. Comput. Phys., 140, 370-399.

[6] Chu, P.C., and C.W. Fan, 1999: A three-point sixth-order nonuniform combined compact difference scheme. J. Comput. Phys., 149, 1-12.

[7] Chu, P.C., and C.W. Fan, 2000: A three-point sixth-order staggered combined compact difference scheme. Math \& Computer Modeling, 32, 323-340.

[8] Chu, P.C., and C.W. Fan, 2001: An accuracy progressive sixth-order finite difference scheme. J. Atmos. Oceanic Technol., 18, 1245-1257.

[9] Chu, P.C., and C.W. Fan, 2002: Hydrostatic consistency in sigma coordinate ocean models. Geophys. Res. Let., submitted.

[10] Ezer, T., H. Arango, A.F. Shchepetkin, Developments in terrain-following ocean models: intercomparisons of numerical aspects. Ocean Modelling, 4, 249-267, 2002.

[11] Foreman, M.G.G., R.F. Henry, R.A. Walters, and V.A. Ballantyne, 1993: A finite element model for tides and resonance along the north coast of British Columbia. J. Geophys. Res., 98, 2509-2532.

[12] Haidvogel, D.B., and A. Beckmann, 1997: Numerical modeling of the coastal ocean. The Sea (edited by K.H. Brink and A.R. Robinson), Vol 10, pp. 457-482, Wiley, New York.

[13] Hermeline, F. 2000: A finite volume method for the approximation of diffusion operators on distorted meshes. J. Comput. Phys., 160, 481-499.

[14] Kantha, L.H., and C.A. Clayson, 2000: Numerical Models of Oceans and Oceanic Processes. Academic Press, San Diego, 940pp.

[15] Kobayashi, M.H., 1999: On a class of Pade finite volume methods. J. Comput. Phys., 156, 137-180.

[16] Le Provost, C., M.L. Genco, F. Lyard, P. Vincent, and P. Canceil, 1994: Spectroscopy of the world tides from a finite element hydrodynamic model. J. Geophys. Res., 99, 2477724797.

[17] Lynch, D.R., J.T.C. Ip, C.E. Naimie, and F.E. Werner, 1996: Comprehensive coastal circulation model with application to the Gulf of Maine. Cont. Shelf Res., 16, 875-906.

[18] Mellor, G.L., T. Ezer, and L.-Y. Oey, 1994: The pressure gradient conundrum of sigma coordinate ocean models. J. Atmos. Oceanic Technol., 11, 1 126-1 134.

[19] Ogura, Y. and N.A. Phillips, 1962: Scale analysis of deep and shallow convection in the atmosphere. J. Atmos. Sci., 19, 173-179.

[20] Song, Y. and D.B. Haidvogel, 1994: A semi-implicit ocean circulation model using a generalized topographyfollowing coordinate. J. Comput. Phys., 115, 228-244. 